\title{
Implementation of Resilience Engineering for Operating Room. Unveiling the Hidden Interactions among Multi-Professionals in a Surgical Team
}

\author{
Takanori Ayabe ${ }^{1,2^{*}}$, Masaki Tomita1, Ryo Maeda1, Manabu Okumura², Kunihide Nakamura ${ }^{2,3}$, \\ Shin Nakajima ${ }^{4}$, Kazue Nakajima ${ }^{5}$ \\ ${ }^{1}$ Division of Thoracic and Breast Surgery, Department of Surgery, Faculty of Medicine, University of Miyazaki, Miyazaki, Japan \\ ${ }^{2}$ Department of Patient Safety Management, University of Miyazaki Hospital, Miyazaki, Japan \\ ${ }^{3}$ Division of Cardiovascular Surgery, Department of Surgery, Faculty of Medicine, University of Miyazaki, Miyazaki, Japan \\ ${ }^{4}$ National Hospital Organization, Osaka National Hospital, Osaka, Japan \\ ${ }^{5}$ Department of Clinical Quality Management, Osaka University Hospital, Osaka, Japan \\ Email: ^tayabe@med.miyazaki-u.ac.jp
}

How to cite this paper: Ayabe, T., Tomita, M., Maeda, R., Okumura, M., Nakamura, K., Nakajima, S. and Nakajima, K. (2020) Implementation of Resilience Engineering for Operating Room. Unveiling the Hidden Interactions among Multi-Professionals in a Surgical Team. Surgical Science, 11, 242256.

https://doi.org/10.4236/ss.2020.119027

Received: August 2, 2020

Accepted: September 14, 2020

Published: September 17, 2020

Copyright $\odot 2020$ by author(s) and Scientific Research Publishing Inc. This work is licensed under the Creative Commons Attribution International License (CC BY 4.0).

http://creativecommons.org/licenses/by/4.0/ (c) (i) Open Access

\begin{abstract}
Background: The implementation of resilience engineering for an operating room is difficult; however, its study would become important for the surgeon's personal and surgical team in order to develop a new surgical safety management. An expert operator must perform an operation with his surgical team that includes an anesthetist, scrub nurse, and young assistant. However, there exist some gaps among these multi-professionals. Objective: From the viewpoint of an expert operator, to have an operation go well, we would describe how to reconcile their gaps. We will explain the gaps among the multi-professionals in a surgical team, such as hidden interactions between the operator and anesthetist, surgeon and scrub nurse, and expert operator and young assistant. Material and Methods: We assumed three types of interactions among the multi-professionals in the operating theater and we clarified how to bridge the gaps by revealing what the operator thinks, what the anesthetist thinks, what the scrub nurse thinks, and what the young assistant thinks in the surgical team, and by understanding how they perform during surgery. Outcomes: What the expert operator thinks and how he performs in surgery is summarized by the following three items: 1) safety is first, 2) achieving the operative purpose, and 3) fast surgery. We interviewed the surgical team members. In order for the surgery to go well, what the important thing is "safety first" for any surgical professionals. The sentence, "safety is first" is the magic words, such as "open sesame". They can communicate with each other on the
\end{abstract}


spot and build the team and system. To develop a strong and resilient surgical team, these four behaviors are important to improve the performance as a system: 1) sharing the same goal and same priority, 2) understanding gaps with clear verbal communication. Coming out from own professional boundaries (takotsubo), speaking in words, 3) all of us, having a strong will (iron heart), and learning anger management, and 4) improvising even in difficult situations. Conclusion: We would like to summarize the items learned from my three described scenes, which are 1) to develop a strong and resilient surgical team, 2) what we have to do, and how we have to perform, and 3) how we can develop a team and system.

\section{Keywords}

Resilience Engineering, Hidden Interactions, Multi-Professionals, Surgical Team

\section{Introduction}

The implementation of resilience engineering for an operating room is difficult; however, its study would become important for the surgeon's personal and surgical team in order to develop a new surgical safety management. In order to perform surgical safety with high-quality surgery, we have to implement resilience engineering for the real world of surgical healthcare.

\subsection{Flow of Surgical Treatment in Lung Cancer}

Surgical practice is individualized treatment tailored to the patient. Lung cancer treatment is combined with surgery, chemotherapy, and radiotherapy according to the patient's condition and the clinical tumor stage while referring to clinical guidelines [1]. In the case of lung cancer found at a relatively early stage, the surgical removal of the tumor is recommended to be the first choice. However, in the case of advanced and unresectable lung cancer, medical treatments other than surgery, that is, chemotherapy and radiation therapy, are the focus. Whether lung cancer can be removed, it is evaluated by a preoperative imaging examination and laboratory findings. Based on the preoperative patient's medical examination (severity of the underlying disease, presence of complications, age, daily activity, etc.) and the clinical stages of cancers, the surgical procedure is determined in consideration of the curability of the cancer and risk of intraoperative and postoperative complications.

For example, in the case of the surgery for right upper lobe lung cancer, the right upper lobe must be removed and the mediastinal lymph nodes must be dissected. Surgery is performed under general anesthesia with the lesioned right lateral chest up. Thoracic surgery is performed by cutting the lateral chest skin (10 - $15 \mathrm{~cm}$ in length) and opening the thoracic wall, then video-assisted thoracic surgery is performed by opening three or four $1-2 \mathrm{~cm}$ long holes in the tho- 
racic wall and inserting a thoracoscope or surgical forceps. The right lung is divided into three parts, that is, the upper, the middle, and the lower lobes. After the pulmonary artery and vein flowing into the upper right lobe and the bronchus of the upper right lobe are dissected, the mediastinal lymph nodes are dissected and the upper lobectomy with mediastinal lymphadenectomy is achieved. After confirming that there is no bleeding and no air leakage from the lungs, the chest drain tube is placed in the chest cavity and the skin is closed, and the entire procedure is completed.

Surgery is performed using the knowledge and skills of the surgeon. However, an unexpected event can happen both expectedly and unexpectedly during the operation. Expectations include tissue adhesions and bleeding. Surgery is performed in response to these conditions, and the surgical procedure is changed and adjusted as needed depending on the findings during surgery and the progress of the surgery aiming for complete excision of the tumor. In the corresponding process and the result, as there is a fine difference between all cases, the same surgery is never carried out.

The surgeon always seeks the best method by considering the balance of the risk of complications during and after surgery, the effect of preserving organ function, and the therapeutic effect of the tumor resection rate, with patient safety first, while proceeding with the operation during surgery.

\subsection{Implementation of Resilience Engineering to Surgery}

Resilience engineering seeks to determine how to create a team that can cope with the situation in which the progress of surgery changes from moment to moment [2]-[8]. As a premise, it is necessary for the members participating in the surgery to share the goal of the team and the image of the operation flow. However, in reality, there is a tendency for the target and surgical image to be misaligned among the members. The key to building an effective team is how to fill this gap and change from "same bed, different dream" to "same bed, same dream" (Figure 1). The surgical team is composed of various occupations such as operator, anesthetist, scrub nurse, and young assistant. Thinking of each occupation as an octopus, each octopus lives in each takotsubo (a silo in English) (Figure 1 and Figure 2). The desired surgery for each image depends on the situation, such as complete tumor removal, safe surgery, and speedy surgery. The octopus cannot enter the other takotsubo, what becomes of in it, is the reality that each person does not know what the others are thinking. Therefore, from the viewpoint of the surgeon, we would like to discuss the consciousness gap that tends to occur between members who live in different takotsubos within the surgical team and how to optimize it.

\section{Material and Methods}

Figure 3 shows that a surgical team consists of an expert operator, young assistant, video-camera assistant, and scrub nurse in the clean field. A circulating 


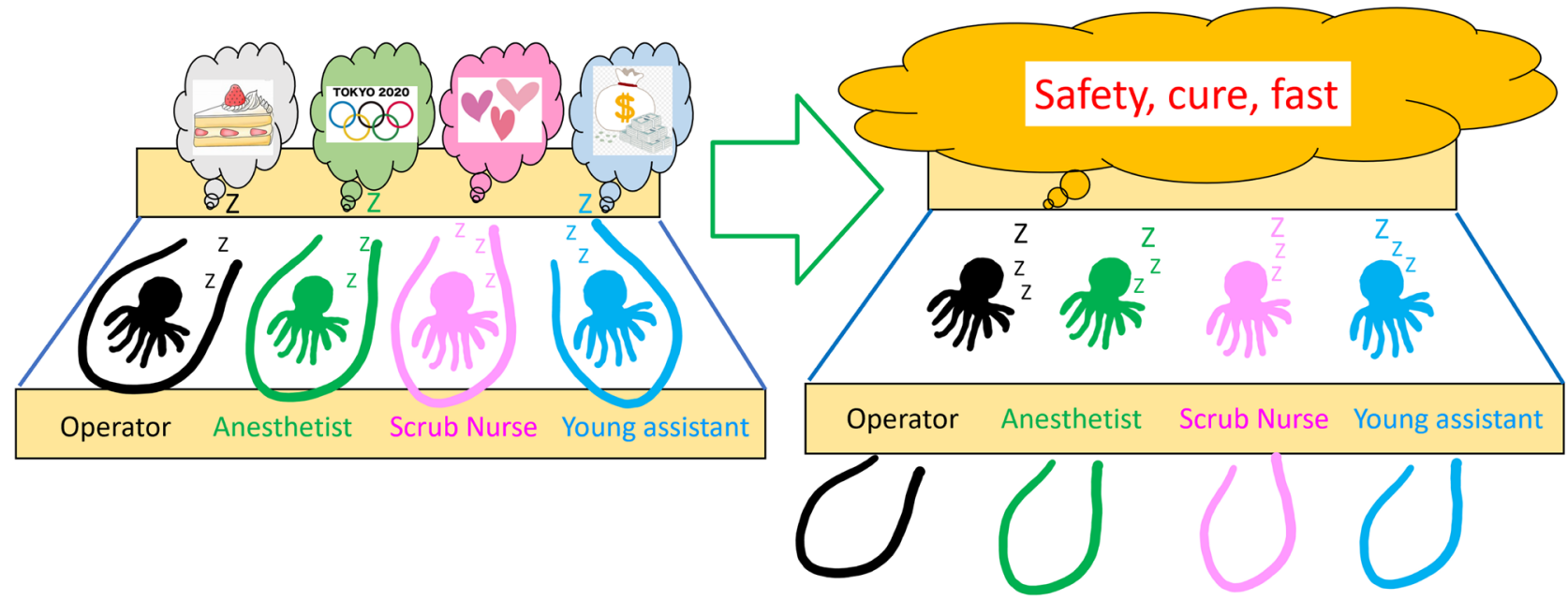

Figure 1. From "Same bed, different dream" to "Same bed, same dream". The left figure shows "Same bed, different dream". Medical staff, each octopus, that is, operator, anesthetist, scrub nurse, and young assistant live in each takotsubo, with different professional boundaries. Thus, each medical staff can't have the same dream. In the operating room, we noted some gaps among the multi-professional interactions. An expert operator, as a leader, must unify this disjointed state. The right figure shows "Same bed, same dream". Each medical staff, octopus have to come out from their own takotsubo. No, I'd rather, they come out on their own, and they have to get some to work. They must have the same dream on the same bed during operation. The dream is, so-called, "safety, cure, and fast" on the multi-professional healthcare bed. However, we have never had the same dream. We have to transform during surgery. Let's go looking for the tricks of realizing the "same dream on the same bed". What we want to do is to realize the three hidden interactions among the surgical members.

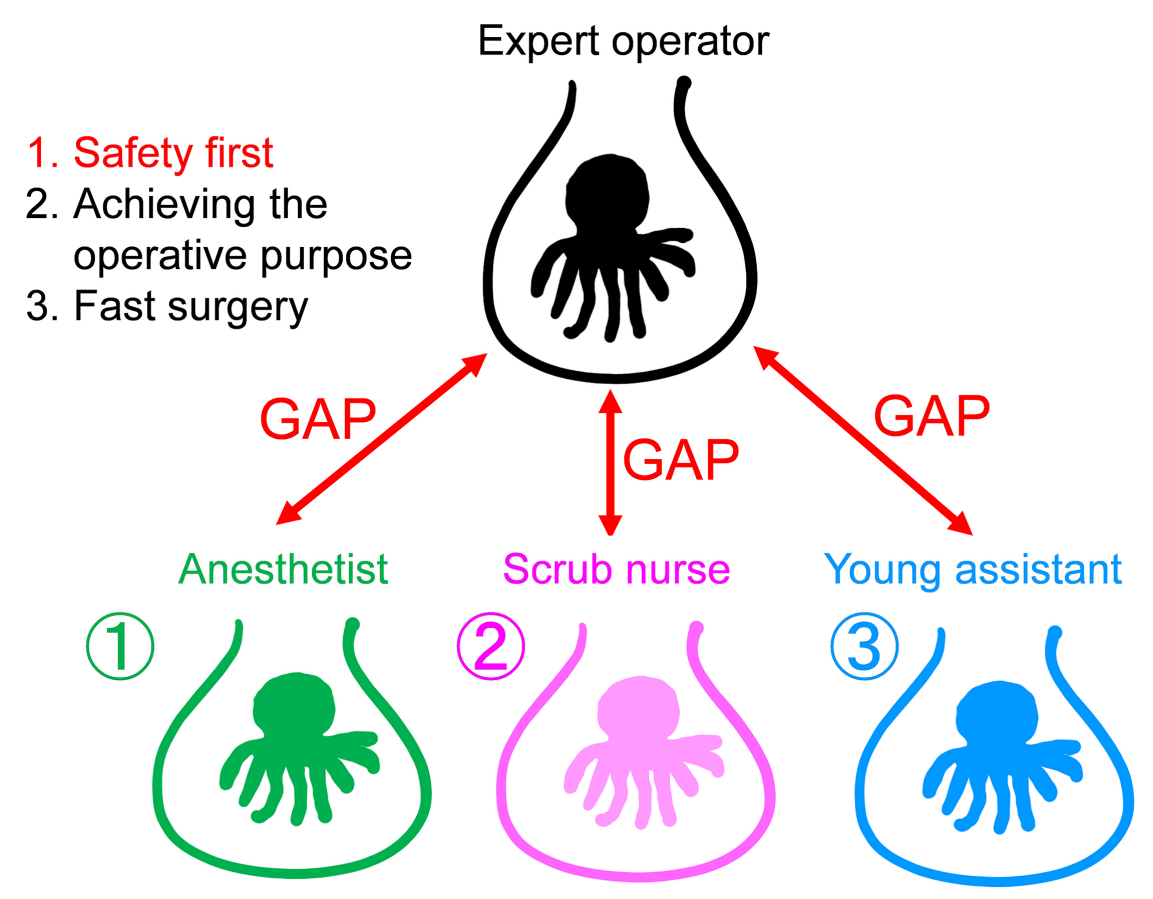

Figure 2. Gaps among multi-professional's "work-as-imagined" and the expert's "workas done". When the expert operator performs surgery with a team, what he thinks and what he performs are, 1) safety is first, 2) achieving of operative purpose, and 3) fast surgery. However, the expert operator feels some gaps among the interactions with surgical multi-professionals. 


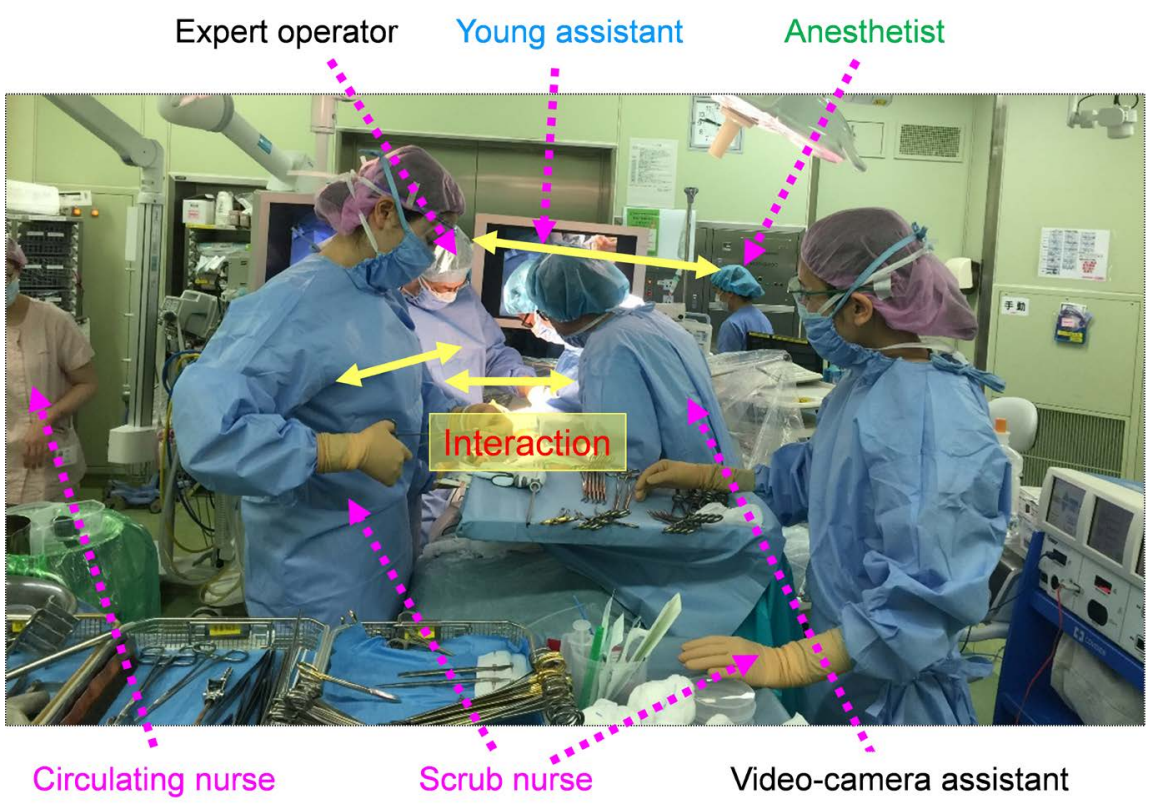

Figure 3. Surgical members in operating theater. A surgical team consists of expert operator, young assistant, video-camera assistant, and scrub nurse in the clean field. A circulating nurse and anesthetist are around but outside the operating field. In the operating filed, three yellow arrows display each interaction with the expert operator and anesthetist, scrub nurse, and young assistant.

nurse and anesthetist are outside of the operating field. In the Figure 3, we would like to explain the hidden interactions among the surgical team; the three yellow arrows display each interaction with the expert operator and anesthetist, scrub nurse, and young assistant. In the surgical operating room, we propose three scenes of interactions among the multi-professionals, that is, we would describe how to reconcile their gaps.

An expert operator must perform an operation with a surgical team that consists of an anesthetist, scrub nurse, and young assistant. However, there exists some gaps among these several-professionals (Figure 2). What the expert operator thinks and how he performs in surgery is summarized in three areas (Figure 2), which are 1) safety is first, 2) achieving the operative purpose, and 3) fast surgery.

We will explain the gaps among the several-professionals in the surgical team, such as hidden interactions between the expert operator, anesthetist, scrub nurse, and young assistant. From the viewpoint of the expert operator, for the operation to go well, we will explain how to reconcile the gaps. In the present study, we will reveal the gaps and hidden interactions among the several-professionals and how to reconcile their gaps.

\section{Result}

\subsection{Composition and Role of Surgical Team Members}

The members of the surgical team are composed of an operator, assistants, anes- 
thetist, scrub nurses, and circulating nurses (Figure 3 ).

The surgeon directs the progress of the operation and performs the main operations during the surgery. Therefore, the operating procedure is set before surgery, the surgical procedure is determined, and the overall procedure is considered. During the operation, one must pay attention to whether or not the operation is smoothly proceeding, and immediately deal with complications, such as bleeding and hypoxemia, and depending on the circumstances, the operation may be stopped or withdrawn.

The assistant is often a young surgeon, and usually two people will enter the surgery. Sometimes a young surgeon is in charge of the surgery for surgical education and training, and an experienced surgeon serves as an assistant.

Scrub nurses, also called instrumentation nurses, wear surgery clothes and enter the surgery, and hand over or use surgical instruments directed by the surgeon or assistant and attach to instruments that have been returned from the surgical field. They have the role of removing blood clots and organizing it so that it can be immediately used. Since surgeons and assistants often perform surgical operations without taking their eyes off the operative field, scrub nurses are required to understand the progress of the surgery and to hand over the required instruments in a timely manner. In some cases, the instruments used in surgery are assembled. In the case of thoracic surgical instruments, they include the thoracoscope, suction device, automatic suture stapler, electrosurgical knife, an ultrasonic coagulation and incision apparatus, fibrin glue sprayer, and similar endoscopic surgical instruments.

The circulating nurse does not wear a garment and assists the scrub nurse from outside the clean operative field, add additional missing surgical instruments to the surgical field, adjust the room temperature, prepare blood transfusions, and make contacts outside the operating room.

The anesthetist has the role of anesthetizing surgical patients to sedation and analgesia and to manage the general conditions such as breathing and blood pressure. In an emergency situation, such as bleeding, the anesthetist must transfuse or administer emergency medicines to stabilize the patient's general vital condition.

Examples of common gaps from the viewpoint of each occupation in the interrelationships between the experienced surgeon and anesthetist, experienced surgeon and scrub nurse, experienced surgeon and young assistant during operations involving multiple occupations as already described, we would like to clarify the method and the hidden interactions to show the gaps.

Hereinafter, each job is explained with the author as the operator and the described gap.

\subsection{Gap in Thinking between Expert Operator and Anesthetist}

Figure 4 shows a gap between the expert operator and anesthetist. Figure 5 shows the hidden interactions to reconcile the gap. 
Expert operator

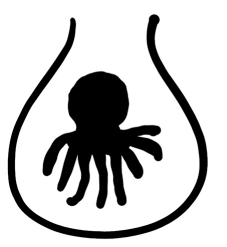

This step must be done slowly, absolutely for safety
Anesthetist
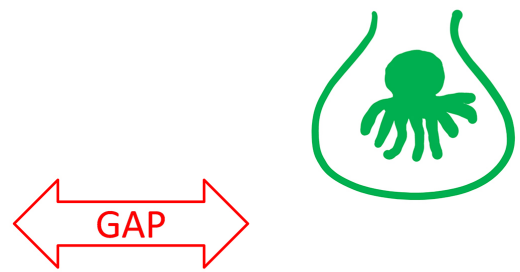

Do operation quickly
(Fast surgery is good)
Next anesthesia is
waiting

Figure 4. The gap between the expert operator and anesthetist. In first scene, "What does the expert operator think and how does he perform?" "This step must be slowly done, because this is an important point." "An unexpected bleeding might happen." "I have to perform the complete operation, absolutely for safety." In contrast, "What does the anesthetist think and how does he perform?" "Do the operation quickly" (My basic priority is based on the fact that the fast surgery is good).

\section{To reconcile the GAP}

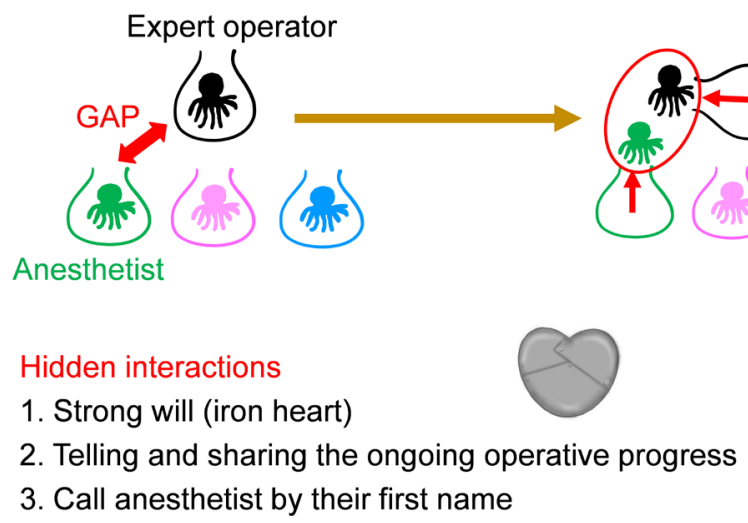

Figure 5. Hidden interactions to reconcile the gap between the expert operator and anesthetist. They live in their own takotsubo, but there is a gap. Removibg the boundaries, and closing the gap are needed. From the expert operator's viewpoint, I found three hidden interactions between them. 1) Strong will (iron heart). Strong mental, and hard spirit. 2) Telling and sharing the ongoing progress of the operation. 3) Call the anesthetist by their first name.

The following is the order in which the surgeon considers prioritizing the surgery.

1) Perform safe surgery and return the patient to outside the operating room without complications.

2) Achieving surgical goals aimed at healing, such as complete resection of malignant tumors.

3) A short time, if possible for the surgery, so as not to burden the members of the surgical team and the patient.

It is important to integrate such safety, purpose achievement, and speed at a high level. Patient safety is a top priority, but it is not the only goal. If patient 
safety is the one and only goal, it is better not to have surgery. Complications occur and life of the patient is at risk, which will be all gone, but the reverse is no longer possible. In addition, it is not the intention of the surgeon to withdraw without resection of the tumor because the safety is too important. Therefore, if a safe and complete tumor resection can be performed, it is considered acceptable even if it takes some time. Of course, if a safe and complete excision can be done quickly, it is satisfactory.

On the other hand, it goes without saying that patient safety is a top priority for the anesthetist. However, when I worked as an anesthetist, I was not very interested in whether or not the malignant tumor could be completely removed, that is, whether or not the surgical goal was achieved. I was thinking that a short-time surgery was a good operation. The anesthetist may be responsible for 2 - 3 anesthesias a day, and if the surgery is completed as planned, the physical burden on the patient is reduced and the next anesthesia can be started with enough time. If the surgeon finishes the operation in a short time and the operation becomes rough, it will take time due to bleeding. In addition, tumors should not be left behind. Even if it takes time, the goal is to make sure that the important points related to the success of the surgery are performed, and it should be treated in a protective manner so as not to bleed when the blood vessels are peeled off, and a complete removal should be sought.

On the other hand, if the anesthetist considers a short-time surgery to be the preferred surgery, there will be a gap in the way of thinking.

In order to fill the gap in the way of thinking with the anesthetist, the following is what the surgeon should do.

1) Have a strong will (iron heart) that the priority will not change no matter what.

2) Report the progress of the operation to the anesthetist in real time.

For example, the anesthetist may be in a situation where the patient has been exfoliated over time, or may be transfused due to difficulty in hemostasis. Share with the entire surgical team, including the physician.

3) Call the anesthetist by their first name.

These are the keys to a smooth operation.

\subsection{Gap in Thinking between of Expert Operator and Scrub Nurse}

Figure 6 shows a gap between the expert operator and scrub nurse. Figure 7 shows the hidden interactions to reconcile the gap.

Most preferred for the operator is to understand the procedure of scrub nurse surgery, one by one without explaining the required surgical instruments to be handed smoothly in surgical advance. The surgeon gives the ambiguous instructions, such as "Take me that" or "Take this out", because the instrument that is needed during the surgery comes to mind in the first place, the feeling of holding the picture and the hand rather than the name. Also, one of the most unbearable things for the surgeon is the fact that the necessary surgical materials 
Expert operator

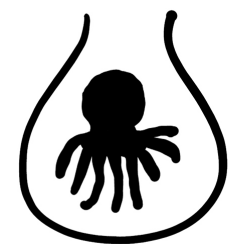

Instrument shortage ?

Unacceptable.

It takes time.

I cannot wait.
Scrub nurse

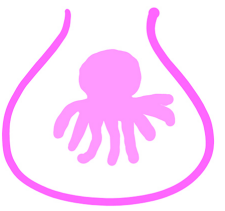

Don't be angry, Don't rush,

My hands cannot move smoothly.

Figure 6. The gap between the expert operator and scrub nurse. In the second scene, "What do they think and perform?", "When necessary equipment has not been prepared." Scrub nurse says "There is no instrument". On the other hand, the operator says "Instrument shortage?", "Unacceptable." "It takes time. I can't wait." In contrast, The scrub nurse says “don't be angry”. "Don't rush." "My hands cannot smoothly move.”

\section{To reconcile the GAP}

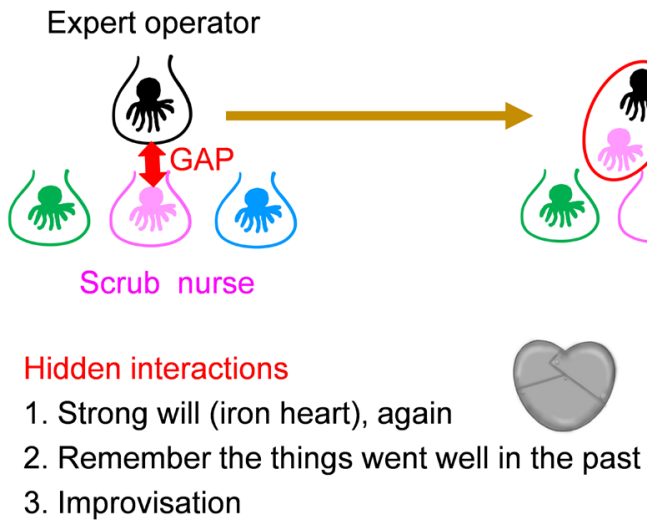

Figure 7. Hidden interactions to reconcile the gap between the expert operator and scrub nurse. From the expert operator's viewpoint, I found three hidden interactions between them. 1) Strong will (iron heart), again. Stronger mental, and harder spirit. 2) Remember that things went well in the past. The operator substitutes similar forceps in a good way for use. Improvisation. Performance adjustment. If the necessary equipment is not available, substitute another similar instrument.

and instruments are not prepared when needed. Still, if it can be found and taken from another operating room, it will be managed, but if there is no surgical material to be prepared anywhere in the hospital, the operation will be interrupted. It is necessary to wait until the article arrives from outside the hospital, and the waiting time is useless. In some cases, the operation itself may be stopped. It can be understood to some extent that the surgeon becomes emotional at such times.

On the other hand, the top priority for the scrub nurses is the same.

1) Patient safety is first. However, if you come in contact with the surgeon's frustration, 
2) I want you to indicate the necessary equipment in words. I don't know "that" or "it".

3) There is helpless thing without even shouting.

We think that we feel that. So what the surgeon should then do.

1) Have a strong will (iron heart) again no matter what happens, and I don't become emotional.

2) Recall the past that survived the difficult situation.

3) Assistant, anesthetist, nurse with the wisdom of making it in time.

\subsection{Gap in Thinking between Expert Operator and Young Assistant}

Figure 8 shows a gap between the expert operator and young assistant. Figure 9 shows the hidden interactions to reconcile the gap.

The ideal form is that a young surgeon usually enters surgery as an assistant, learns the technique from an experienced surgeon, and gradually grows. Even as an expert surgeon, I think in my heart that if the young person provides a smooth assistance, I will leave it to a young assistant for the next surgery. However, if the doctor cannot be served as a good assistant, the surgeon will think in the following ways:

1) Your hands are in the way and I can't see well. Hold firmly and pull.

2) Do it without saying anything. Why can't you do that?

3) Did you study properly before surgery?

Although it is best, young surgeons cannot suddenly improve because they yell. Now, there is no choice but to have a young surgeon in front of him to help her.

On the other hand, I also experienced as an assistant when I was young,

1) I want you to tell me exactly what I should do. When bellowed, I can't do well.

2) I want to improve my surgery.

Expert operator

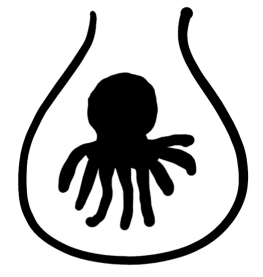

I can't see it.

Don't resist.

Hold on tight.

Why can't you

do that?

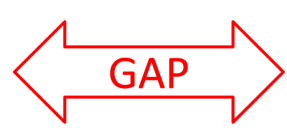

Young assistant

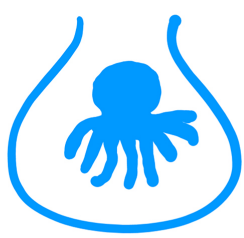

Don't be angry.

Please say what you

want me to do.

I need a proper

instruction.

Figure 8. The gap between the expert operator and young assistant. In the third scene, "What does the expert operator think and perform during the operation?" The expert operator says "I can't see the target". "Don't resist. Hold on tight." "Why can't you do that?" In contrast, "What does the young assistant think and perform?" “'Don't be angry." "Please say what you want me to do." "I need proper instruction." 


\section{To reconcile the GAP}

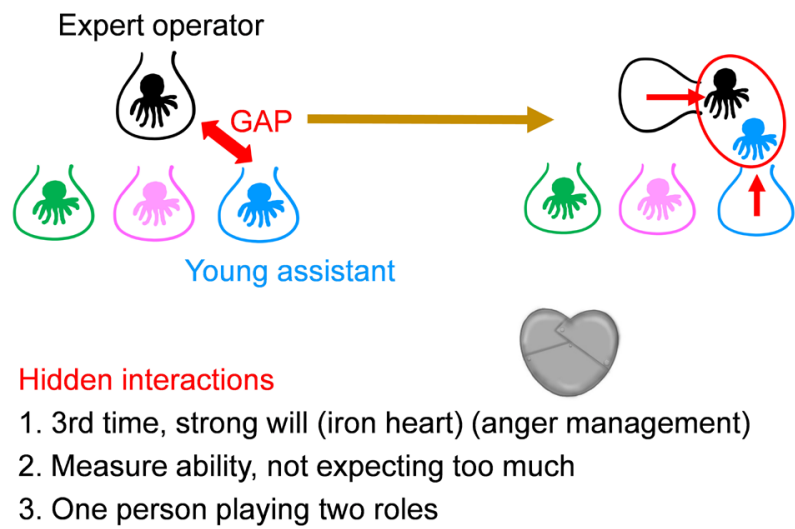

Figure 9. Hidden interactions to reconcile the gap between the expert operator and young assistant. From the expert operator's viewpoint, I found three hidden interactions between them. 1) For the third time, strong will (iron heart), and anger management. The strongest mental, and the hardest spirit. 2) Measure ability, not expecting too much. 3) One person playing two roles. Showing technique, letting him do it, and the operator must always praise him.

I remembered thinking that way when I was young.

The way surgeons think about these two surgeons and how to close the gap is,

1) Have a strong will (iron heart) three times. In other words, it is anger management.

2) Have an assistant who can determine the ability of a young surgeon.

3) The surgeon will show you what the assistant should do. Carefully teach your assistant during the surgery as the best educational opportunity.

\section{Discussion}

\subsection{How to Reconcile the Gaps in Order to Have a Successful Operation as a Surgical Team}

Figure 10 shows the best methodology for a professional surgical team. Figure 11 shows the importance of performance improvement as a system in order to provide resilient surgical team.

Bridging the gap between occupations, it was mentioned at the beginning that the key to creating an effective surgical team is how to change from "same bed, different dream" to "same bed, same dream" (Figure 1). Expert surgeons, anesthetist, scrub nurses, and young assistants, who have different ideas each because they have their own way, it's no wonder that gaps arise. However, in order to smoothly proceed, the hidden interaction between the members of the surgical team and between occupations, we have to understand and respond to gaps. This is team building and resilience implementation [9].

The author asked, "Three things that are important for a successful surgery. What if I get it?" I interviewed expert surgeons, anesthetist, scrub nurses, and young assistants who are multi-disciplinary surgical team members. 


\section{The magic words for the same dream}

1. Safety first
2. Achieving the
operative purpose
3. Fast surgery
$\begin{array}{ll}\text { 1. Safety first } \\ \text { 2. Lookahead of } \\ \text { operative flow }\end{array}$
$\begin{array}{ll}\text { 1. Sain control } \\ \text { 3. Smooth support for } \\ \text { surgery }\end{array}$

Figure 10. The magic words for the same dream. I interviewed the surgical team member. The question is "in order for the surgery to go well", "what is important?", "show three." They answered "safety first" for all surgical professionals. The sentence, "safety is first" is the magic phrase, such as "open sesame", multi-professional octopuses are getting out from their takotsubo. They can communicate with each other on the spot and improve the team and system.

\section{Conclusions}

Development of resilient surgical team Performance improvement as a system

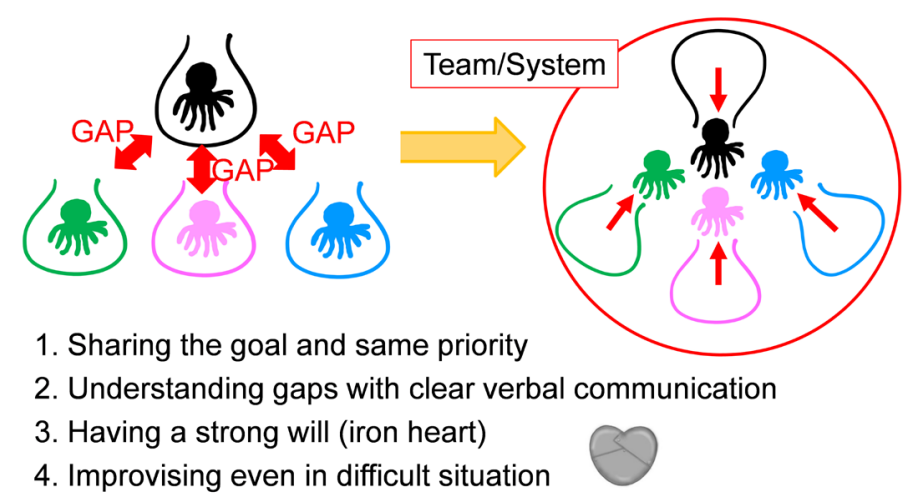

Figure 11. Conclusions. Development of resilient surgical team. Improvement of performance as a system.

Both the expert surgeons and the young assistants then gave the following three responses.

1) Patient safety,

2) Achievement of surgical purpose,

3) Fast surgery.

On the other hand, the anesthetist gave the following three responses:

1) Patient safety,

2) Pain management,

3) Support for smooth surgery.

The scrub nurse gave the following three responses:

1) Patient safety, 
2) Understand the flow of surgery,

3) Quick response to surgeon's instructions.

After all, all members of the surgical team consider "Patient safety" the most important factor. Also, the second and third most important elements are to fulfill their respective responsibilities. However, I found that I was working more professionally.

Considering the above results comprehensively, i.e., the surgical team members improve their performance by successfully forming a resilient surgical team.

All you need to do is,

1) Share target priorities among the members.

2) Understand gaps between the various occupations and clearly communicate in words.

3) Everyone, including the surgeon, has a strong will (iron heart).

4) Improving the difficult situation through improvisation.

Overall,

The important factors differ depending on the occupation, but the practice is common.

\subsection{Differences in Thinking between the General Patients and the Surgical Team (Surgical Provider Side)}

Figure 12 shows a future prospect in order to effectively improve our surgical team.

Finally, we would like to touch on the differences in thinking between the general patients and the surgical team. The general patients and family members may have imagined that a multi-disciplinary surgical team has gathered before the surgery to consider the surgical method for each case at the conference. However, in most cases, the assistant asks the surgeon about the surgical policy before surgery, and the surgeon can easily say two or three words and finish the meeting by only answering. Regarding the diagnosis and treatment policy of

\section{Future prospect}

To reveal another hidden interactions among surgical team

To effectively develop our team:

1. Learning while doing it

2. Doing what you've learned on the spot

3. All member, having the above attitude

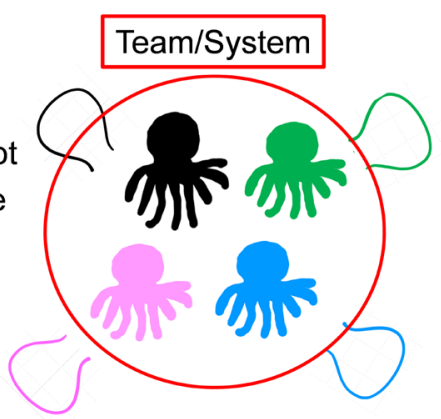

Figure 12. Future prospect. To effectively develop our team and what I want to keep is 1) learning while doing it, 2) doing what you've learned on the spot, and 3) all member, having the same attitude. 
lung cancer itself, radiologists and respiratory physicians may jointly hold conferences. It is best to do the surgical treatment whether the treatment is radiotherapy or chemotherapy. This is not a discussion about the method of surgery.

In addition, the general patients and families may expect that many occupations gather after the surgery to examine the results of each surgery. Unfortunately, that is not the case. I have never experienced postoperative conferences that exceeded my occupation so far. Do not hold a conference, only a short discussion. Another surgeon who had experienced the same situation about this point that did not go as expected in the surgery, "In this case, I survived". "It was good", and the usual pattern is that all of them ask "I see."

In such a situation, there is no other way than to perform the examination beyond the job type on the spot, that is, during the operation. For example, a newcomer can perform surgery as a scrub nurse. If the newcomer is proficient in handling various surgical instruments (thoracoscope, suction device, automatic amputation suture device, electrosurgical unit, ultrasonic coagulation incision device, fibrin glue sprayer, endoscopic surgical instruments, etc.), and if not, you should find free time during the operation and learn and practice assembly and handling on the spot under the guidance of a young assistant surgeon or expert nurse. Don't expect to spend time practicing after surgery. The next surgery just stumbles in the same place. On the other hand, new nurses learn various things even during surgery, and have to be prepared for it on the spot and have surgery. You should be able to learn various things effectively if you prepare your mind and undergo surgery.

We describe the important points of the present study,

1) Intraoperative teamwork is a dynamic process through the interaction of the surgeon with the other surgical team members.

2) Resilient team performance during disturbances and constraints requires sharing of the target priorities among the team members, communication, stress management, and improvisation.

3) In order to effectively develop a surgical team, it is necessary to 1) learn while doing it, 2) do what you have learned on the spot, and 3) have the attitude of all members of the surgical team.

\section{Conclusions}

By clarifying the exchange of silent messages between the surgical team members, the performance of the team can be gradually improved, and the safety of the surgery, completion of the objective, shortening of the surgical time, etc. are expected to gradually improve. As a future resilience engineering study, the author wants to explore how surgical teams can efficiently grow while revealing the silent interactions that still exist in surgical teams.

The first thing to start with 1) learn while doing, 2) do what you learn on the spot, and 3) all members of the surgical team have the attitude described above. We would like to find a new method for a resilient healthcare. 


\section{Acknowledgements}

This study was supported by JSPS KAKENHI Grant Number JP18K04636. This study was approved by the University of Miyazaki Hospital Clinical Research Ethics Board (No. C-0099) in June 2020.

\section{Conflicts of Interest}

The authors declare no conflicts of interest regarding the publication of this paper.

\section{References}

[1] (2018) NCCN Clinical Practice Guidline in Oncology, Non-Small Cell Lung Cancer. Version 2. https://www2.tri-kobe.org/nccn/guideline/lung/english/non small.pdf

[2] Hollnagel, E. and Braithwaite, J. (2013) Resilient Health Care (Ashgate Studies in Resilience Engineering). CRC Press.

[3] Hollnagel, E., Paries, J. and Wreathall, J. (2013) Resilience Engineering in Practice: A Guidebook (Ashgate Studies in Resilience Engineering). CRC Press.

[4] Hollnagel, E., Braithwaite, J. and Wears, R.L. (2018) Delivering Resilient Health Care. Routledge. https://doi.org/10.4324/9780429469695

[5] Braithwaite, J., Wears, R.L. and Hollnagel, E. (2019) Resilient Health Care, Volume 3: Reconciling Work-as-Imagined and Work-as-Done. CRC Press.

[6] Wears, R.L. and Hollnagel, E. (2015) Resilient Health Care, Volume 2: The Resilience of Everyday Clinical Work (Ashgate Studies in Resilience Engineering). CRC Press.

[7] Woods, D.D. and Hollnagel, E. (2006) Resilience Engineering: Concepts and Precepts. CRC Press.

[8] Nemeth, C.P. and Hollnagel, E. (2014) Resilience Engineering in Practice, Volume 2: Becoming Resilient (Ashgate Studies in Resilience Engineering). CRC Press.

[9] Edmondson, A.C. (2012) Teaming: How Organizations Learn, Innovate, and Compete in the Knowledge Economy. Jossey-Bass. 